\title{
Rogue waves, rogue events and extreme wave kinematics in spatio-temporal fields of simulated sea states
}

\author{
A. Sergeeva ${ }^{1,2}$ and A. Slunyaev ${ }^{1,2}$ \\ ${ }^{1}$ Institute of Applied Physics, Nizhny Novgorod, Russia \\ ${ }^{2}$ Nizhny Novgorod State Technical University, Nizhny Novgorod, Russia \\ Correspondence to: A. Sergeeva (a.sergeeva@hydro.appl.sci-nnov.ru)
}

Received: 10 January 2013 - Published in Nat. Hazards Earth Syst. Sci. Discuss.: 29 January 2013

Revised: 18 May 2013 - Accepted: 23 May 2013 - Published: 9 July 2013

\begin{abstract}
An approach to the extensive study of rogue wave occurrence in numerical simulations is presented. As a result of numerical simulations of the unidirectional wave evolution, spatio-temporal fields of wave data of the size $20 \mathrm{~min} \times 10 \mathrm{~km}$ are obtained with high resolution in time and space and are used for statistical analysis with the focus on extreme waves. Having the exhaustive information on the wave evolution enables us to capture the detailed picture of individual rogue waves; to detect intermittent rogue wave events, which last for a significantly longer time, and hence, to depict the portrait of a rogue wave. Due to the benefit of having full-wave data, the question of relation between extreme wave kinematics and extremely high waves is discussed in the statistical sense.
\end{abstract}

\section{Introduction}

The rogue (or freak wave) topic is of very high interest nowadays in marine engineering and geophysical communities. The huge oceanic waves seem to come from nowhere and to disappear with no trace, but at the transient moment of their occurrence they cause ultimate impact, damage and destroy boats and sea platforms, and kill people. Rogue wave phenomenon challenges physical and mathematical researches, which are now numerous, see reviews in (Kharif et al., 2009; Dysthe et al., 2008; Slunyaev et al., 2011). Though main physical mechanisms which can cause these waves seem to be formulated, realistic sea waves represent a quite complex object for investigation, so that the very vital problems: what rogue waves look like, how long they live and how probable they are to occur have not been answered with certainty so far.
Derivation of rogue wave probability from the primitive water wave equations is too complicated: the usual assumptions of the kinetic theory are violated, and wave phase coherence and strong nonlinear effects must be taken into consideration. Direct measurements of sea waves have now resulted in an impressive amount of data. However, rogue waves seem to produce extreme effects on measuring devices, so that the number of reliable recorded time series of rogue waves turns out to be insufficient to build the statistics of in situ rogue waves (Christou and Ewans, 2011), which often leads to conflicting conclusions of different studies. Besides, the statistical ensemble must consist of homogeneous data; this requirement is difficult to fulfill when processing in situ records.

Laboratory and numerical simulations provide an efficient tool for studying stochastic wave evolution. Such experiments have been performed by many authors (e.g., numerical studies by Onorato et al., 2001, 2002; Dysthe et al., 2003; Janssen, 2003; Socquet-Juglard et al., 2005; Chalikov, 2005, 2009; Gramstad and Trulsen, 2007; Annenkov and Shrira, 2009; and laboratory experiments by Onorato et al., 2005, 2009; Mori et al., 2007; and many others), including the authors of the present study, see numerical simulations (Pelinovsky and Sergeeva, 2006; Shemer et al., 2010b; Slunyaev and Sergeeva, 2011) and laboratory studies (Shemer and Sergeeva, 2009; Shemer et al., 2010a, b). The simulations performed in the present research use a similar arrangement. A random superposition of sinusoidal waves with prescribed spectral shape is used as the initial condition at $t=0$ and simulated in time. Then, the result of wave evolution is analyzed. We emphasize that the wave system may undergo essential statistical evolution, which yields violation of the stationarity 
assumption, see discussion in (Shemer et al., 2010a, b; Slunyaev and Sergeeva, 2011).

Even when detailed information on wave dynamics is potentially available (for example, in stochastic numerical simulations), in most of the studies only a small amount of wave information is processed. For example, in the absolute majority of laboratory experiments, the data consists of time series of surface elevation recorded by a few gauges. In the vast majority of the numerical simulations, rogue waves are analyzed only at the moment when the maximum wave height is attained. The wave field data normally cannot be stored with too high a frequency because this would slow down the simulation gradually, and would increase the amount of data. Meanwhile, the simulation of the initial problem employs integration in time, thus a fine time resolution of wave dynamics is naturally available.

If a rogue wave occurs (let us assume that the wave does not overturn, so that the simulation can be continued), it possesses a finite lifetime, and it is a rogue wave at every moment of this time interval. We emphasize this obvious fact and analyze all the moments of the freak wave evolution. To produce one realization of a stochastic wave dynamics, we simulate quasi-random waves in time, and the simulated data is stored with a sufficiently high frequency for further analysis. Besides the surface elevation, velocity fields are also collected through the evolution, thus, the full wave data is available for the analysis.

Velocities and accelerations which are experienced by the fluid due to intense wave motions are much less investigated. Papers by Grue et al. (2003), Grue and Jensen (2006, 2012) combine theoretical/numerical and laboratory (by PIV methods) investigations, and contain important findings (see other references on direct measurements of wave kinematics therein). Different wave sequences (focusing and random, unidirectional and directional waves) were considered in those publications. In the present work we are focused on the comparison between the kinematics of very intense (rogue) waves and "ordinary" waves.

Usually, 20 min time series represent the in situ measurements, therefore the time interval of $20 \mathrm{~min}$ is considered in the present study. To ensure that all the waves which pass one point in space during the $20 \mathrm{~min}$ period are indeed different, we take a sufficiently long spatial domain of simulations: $C_{\mathrm{gr}} \times 20 \mathrm{~min} \approx 10 \mathrm{~km}$, where $C_{\mathrm{gr}}$ is the typical group velocity. The data grid consists of $2048 \times 2048$ points, which corresponds to about $5 \mathrm{~m} \times 0.6 \mathrm{~s}$ mesh size. The moderate level of discretization is chosen with the purpose of analyzing the data employing an ordinary PC; of course, a finer mesh in time and/or in space may be produced.

The spatial domain contains about 60 individual waves; their evolution is considered for about 120 wave periods (2048 instants). Twenty realizations of irregular waves for each initial condition, specified by the spectrum shape (the sea condition), build up the statistical ensemble, which results in about 2500000 individual waves in the spatial do- main (20 realizations $\times 60$ waves $\times 2048$ instants). All these waves are used for the analysis. It is important to be aware of a direct comparison between the statistical results obtained in this paper and the statistics based on measurements in one point. In our case not all the waves are statistically independent; this issue will be discussed below in Sect. 3.1. The reverse side of the present approach is that if a rogue wave appears, then it is examined at all phases of its evolution. Possessing both the spatial and temporal data, the dynamics of a rogue wave are considered in Sect. 3.2, in particular, the lifetime and prevailing shapes of rogue waves are obtained. We introduce the concept of a rogue event, which incorporates shorter-living extreme waves, which overpass the formal height criterion on a rogue wave with brief interruptions. With the use of the wave velocity data, collected in our simulations, in Sect. 4 we discuss a very important issue of the wave-structure interaction: how extreme velocities in the simulated wave fields correlate with velocities, culminated during the rogue wave lifetime. The paper ends with conclusions.

\section{The approach for producing space-time wave data sheets}

\subsection{Numerical model}

The primitive equations of potential hydrodynamics for the surface displacement $\eta(x, t)$ and the surface velocity potential $\Phi(x, t)=\phi(x, t, z=\eta)$ have the form

$$
\begin{aligned}
& \frac{\partial \eta}{\partial t}=-\frac{\partial \Phi}{\partial x} \frac{\partial \eta}{\partial x}+\left(1+\left(\frac{\partial \eta}{\partial x}\right)^{2}\right) \frac{\partial \phi}{\partial z} \\
& \frac{\partial \Phi}{\partial t}=-g \eta-\frac{1}{2}\left(\frac{\partial \Phi}{\partial x}\right)^{2}+\frac{1}{2}\left(\frac{\partial \phi}{\partial z}\right)^{2}\left[1+\left(\frac{\partial \eta}{\partial x}\right)^{2}\right],
\end{aligned}
$$

$$
\frac{\partial^{2} \phi}{\partial x^{2}}+\frac{\partial^{2} \phi}{\partial z^{2}}=0, z \leq \eta
$$

$$
\frac{\partial \phi}{\partial z} \rightarrow 0, z \rightarrow-\infty
$$

Here $x$ is the spatial coordinate along the wave propagation, and $t$ is the time variable. The $O z$ axis is directed upward, and $g$ is the gravity acceleration. Equation (3) is the Laplace equation for the velocity potential $\phi(x, z, t)$, Eqs. (1) and (2) constitute the surface boundary condition, and Eq. (4) is the bottom condition for it (an infinitely deep water case is considered).

In this research Eqs. (1)-(4) are integrated by means of a high-order spectral method (HOSM), see (Dommermuth 
Table 1. Parameters of the sea states.

\begin{tabular}{lllll}
\hline Series code & A & B & C & E \\
\hline$T_{0}, \mathrm{~s}$ & $\mathbf{1 0}$ & 10 & 10 & $\mathbf{1 0 . 5}$ \\
\hline$H_{\mathrm{s}}, \mathrm{m}$ & $\mathbf{3 . 5}$ & 7 & 9 & $\mathbf{7}$ \\
\hline Peakedness, $\gamma$ & $\mathbf{3}$ & 3 & 3 & $\mathbf{3 . 3}$ \\
\hline
\end{tabular}

and Yue, 1987; West et al., 1987) with the 4th order RungeKutta integration in time. Strictly speaking, the HOSM is not a fully-nonlinear, but a strongly nonlinear algorithm, which resolves the wave-wave interactions up to the predetermined order $M$ (for $M=3$ the HOSM approach has the same accuracy as the Zakharov equation (Zakharov, 1968)). In the present study parameters $M=3$ and $M=6$ are used for different simulations; the HOSM formulation of West et al. (1987) is employed. Reviewing high-order spectral methods, Tanaka (2001) showed consistency of the approach by West et al. (1987); an inaccuracy of the formulation of Dommermuth and Yue was observed in long-term simulations by Clamond et al. (2006). Simultaneously, the case $M=6$ was shown to correspond to a nearly fully nonlinear case, see the discussion in (Clamond et al., 2006).

\subsection{Initial conditions and preparation of the sea state}

The initial condition for the simulations corresponds to a random superposition of sinusoidal waves with spectrum of the JONSWAP shape

$S(\omega)=\alpha\left(\frac{\omega}{\omega_{0}}\right)^{-5} \exp \left[-\frac{5}{4}\left(\frac{\omega}{\omega_{0}}\right)^{-4}\right] \gamma^{\exp \left[-\frac{1}{2 \Delta^{2}}\left(\frac{\omega-\omega_{0}}{\omega_{0}}\right)^{2}\right]}$

$\Delta(\omega)=\left\{\begin{array}{cc}0.07, \omega<\omega_{0} \\ 0.09 \omega>\omega_{0}\end{array}\right.$.

Parameters of the sea states are specified in Table 1. There $T_{0}$ is the wave peak period, $H_{\mathrm{S}}$ is the significant wave height, and $\gamma$ is the peakedness. Parameter $\alpha$ in (5) is determined through the characteristic wave height, and the angular frequency relates to the wave period as $\omega_{0}=2 \pi / T_{0}$. In this paper the majority of presented results concerns cases A and E. The first one is considered as a low sea state, while the second one corresponds to severe conditions.

Two functions at $t=0$ are required to specify the initial condition for the simulation: the surface elevation, $\eta(x$, $t=0)$, and the surface velocity potential, $\Phi(x, t=0)$. Due to the nonlinearity, these fields are in fact related in a non-trivial way, so that in the case of significant wave nonlinearity and broad spectrum it is very difficult to find the initial condition, which would be a realization of irregular waves with the proper relation between functions $\eta(x, t=0)$ and $\Phi(x$, $t=0)$.

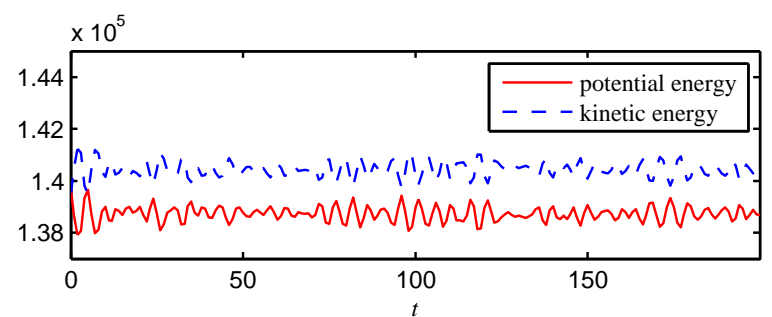

a)

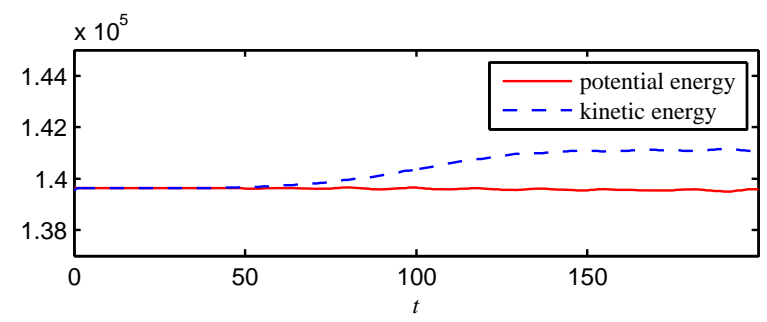

b)

Fig. 1. Potential and kinetic energy of waves in the numerical simulations. Case (b) corresponds to a slow switching on the nonlinearity in the governing equations, while case (a) shows the result of simulation of the original HOSM. Sea state A.

Frequently, one of the fields is taken in the form of a linear superposition of Fourier harmonics with random phases, and the second one is obtained according to the linear relation between wave elevation and velocity. The result of such careless production of the initial condition is displayed in Fig. 1a, where the evolution of potential, $E_{\mathrm{p}}$, and kinetic, $E_{\mathrm{k}}$, energies of the wave system is given, where

$E_{\mathrm{k}}=\frac{1}{2} \int \frac{\partial \eta}{\partial t} \Phi d x, E_{\mathrm{p}}=\frac{1}{2} g \int \eta^{2} d x$.

According to the linear theory, the portions of kinetic and potential energy are equal at the initial moment. This balance is not retained by nonlinear waves. As a result, parasitic counter waves are naturally excited in the course of evolution of the improper initial condition. They are well seen in the $(\omega, e)$ Fourier diagrams of the wave evolution (see Slunyaev and Sergeeva, 2012), and also may be revealed due to the oscillations, which are readily seen in Fig. 1a. Similar oscillations with the period of a half-dominant wave period are typical for the case of standing waves (in other words: existence of two opposite wave systems).

To avoid this undesired effect in the simulations, the nonlinear parts of Eqs. (1)-(4) were turned on slowly to enable the waves to adjust to the nonlinearity normally. The dependences of potential and kinetic energies in this statement are shown in Fig. 1b. The time interval shown in Fig. 1 corresponds to about 20 wave periods, while the typical timescale of this adjustment is one wave period. A smooth departure of the kinetic and potential parts of energy is observed. Simultaneously, the amount of counter waves diminishes gradually. 
Generation of the initial condition in the form of a superposition of sinusoids with random phases implicitly implies the assumption of Gaussian wave statistics. Besides establishing the correct ratio between the kinetic and the potential energies due to generation of proper phase-locked bound waves, the initial stage of wave evolution may undergo a longer transition process, which is related to the forming of natural nonlinear wave groups. This effect is primarily due to the four-wave interactions, and has a typical duration of order $\varepsilon^{-2} T_{\mathrm{p}}$ (where $\varepsilon$ is the wave steepness). This effect has been observed many times in laboratory and numerical experiments, see among others (Shemer and Sergeeva, 2009, 2011; Shemer et al., 2010a, b; Dysthe et al., 2003; Janssen, 2003; Onorato et al., 2005; Annenkov and Shrira, 2009). A relatively simple theory, based on modified nonlinear Schrodinger equation (see, e.g., in Trulsen, 2006), is often able to capture the nonlinear effects at this scale. However, its application may be limited due to the requirements of a narrow spectrum and a weak nonlinearity.

For a given wave intensity, this transition process occurs in the case of sufficiently narrow spectrum and is characterized by a temporal spectrum widening with subsequent partial relaxation. During the transition stage the probability of extreme waves increases greatly. After this acute stage a quasistationary state is attained. Such a process is most pronounced in the case of large values of the Benjamin-Feir Index, which may happen when the JONSWAP spectrum is characterized by a large value of peakedness, $\gamma$. The transition process is not appreciable if the waves are weakly nonlinear or the spectrum is wide (BFI is small). The quasistationary condition is achieved within a few units of the "nonlinear time" $\sim \varepsilon^{-2} T_{\mathrm{p}}$.

In our numerical simulations the first $200 \mathrm{~s}$ of the wave evolution are allocated for the transient processes and are not used for the analysis. The wave system is assumed to be the proper sea state for $t>200 \mathrm{~s}$.

Figures 2 and 3 show the spectra which characterize the obtained sea states A and E correspondingly. These Fourier spectra are averaged; they are built on the basis of all simulated data using 20 realizations. Dashed curves in the figures indicate the corresponding JONSWAP spectrum, which is used for the generation of the initial condition.

It may be noted that the obtained spectrum is somewhat distorted due to the nonlinear processes and moderate number of realizations used for the averaging. However, the agreement between the modeled and the obtained spectra is good. A significant variation of the wavenumber spectrum in time was not observed, which confirms the closeness of the simulated sea states to stationary conditions.

A peak frequency downshift may be seen for the steeper sea state in Fig. 3, which is a well-known effect. Besides, some faster decay of the simulated spectrum tail, in comparison with the JONSWAP curve, is noticeable in Fig. 3. This effect is due to the effective high-frequency wave dissipation, which was introduced to damp the processes of wave breaking in the way similar to (Chalikov, 2005). Otherwise, too

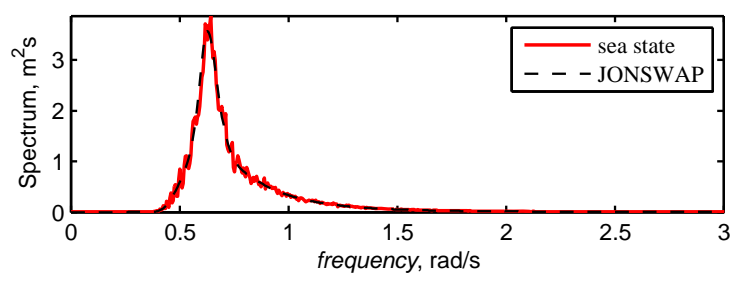

a)

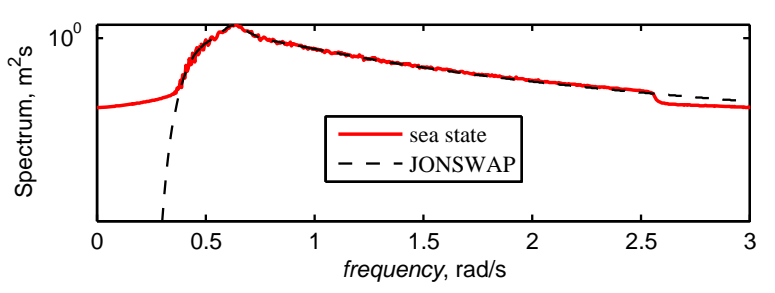

b)

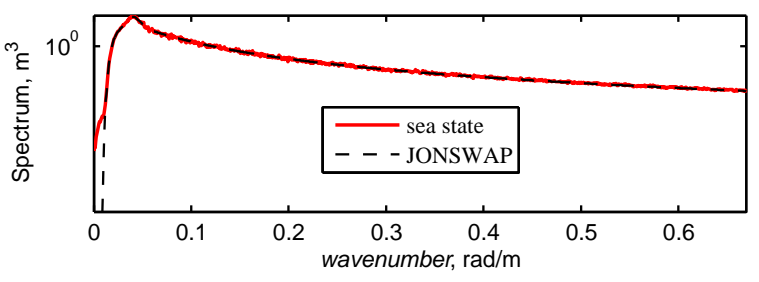

c)

Fig. 2. Averaged frequency spectrum in linear (a) and semilogarithmic (b) axis, and also the wavenumber spectrum in semilogarithmic axis (c) for the sea state A. Dashed curves show the modeled JONSWAP spectrum.

steep waves, which appear during the wave evolution, cause numerical instabilities, and the simulation blows up quickly. Thus, the desired sea state $\mathrm{E}$ is not reproduced in full, and the sea state corresponds to a slightly different condition of sea wave evolution.

The HOSM is unable to resolve very steep waves; it also suffers from instabilities that may normally reduce its capability of long-time simulations. High-intensity waves in case E and the slowly decaying spectrum tail of the JONSWAP function provide favorable conditions for the occurrence of very steep waves. Sometimes the employed highfrequency damping filter was not able to prevent the occurrence of small-scale instability. Then the simulation was interrupted, the steep wave was artificially removed from the field, and the simulation was continued. As a result of the introduced damping and removal of too many sharp waves, the root mean square of surface elevation, $\sigma$, might decrease during the simulation to about $23 \mathrm{~min}$ in total, or up to about $10 \%$. 


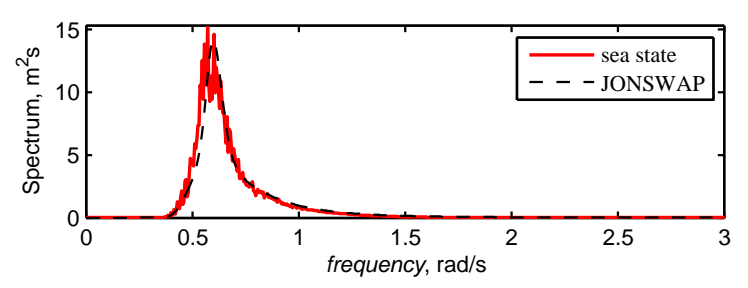

a)

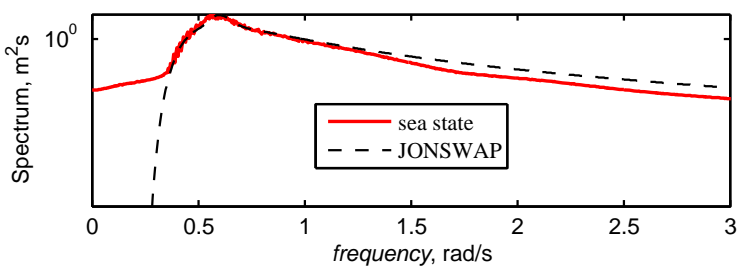

b)

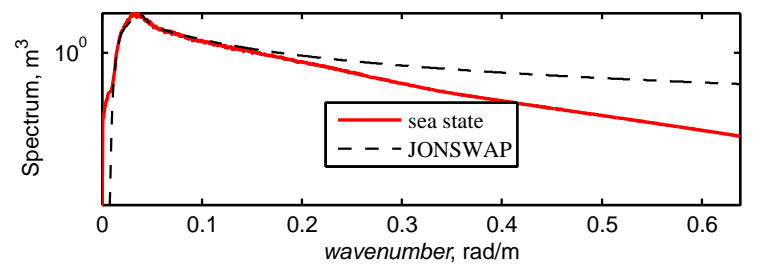

c)

Fig. 3. Averaged frequency spectrum in linear (a) and semilogarithmic (b) axis, and also the wavenumber spectrum in semilogarithmic axis (c) for the sea state E. Dashed curves show the modeled JONSWAP spectrum.

\section{Analysis of the spatio-temporal surface elevations}

The simulated spatio-temporal wave fields of the surface elevation with high resolution in time and space give the comprehensive information about the wave's shapes, and make possible a study of rogue wave's dynamics in detail. Since the considered spatial domain is large (about $10 \mathrm{~km}$ ), the probability of extreme waves in a certain time interval increases in comparison with the probability calculated for time records in a given spatial point. Thus, almost every simulated realization contains several abnormal waves, while the linear theory for the Gaussian sea predicts such a wave to occur in a single point once during about $8 \mathrm{~h}$ (Kharif et al., 2009).

The widely used criterion for rogue wave definition is based on the amplification factor, $A I$ (abnormality index), formulated in terms of the wave height, $H$. The simple common definition of a rogue wave reads

$A I>2, A I=\frac{H}{H_{\mathrm{S}}}$.

Here $H_{\mathrm{s}}$ is the significant wave height determined as the average value of one-third of the highest waves in a record. For the Gaussian sea this parameter could be written with the use of the root mean square of surface elevation as $H_{\mathrm{s}} \approx 4 \sigma$.
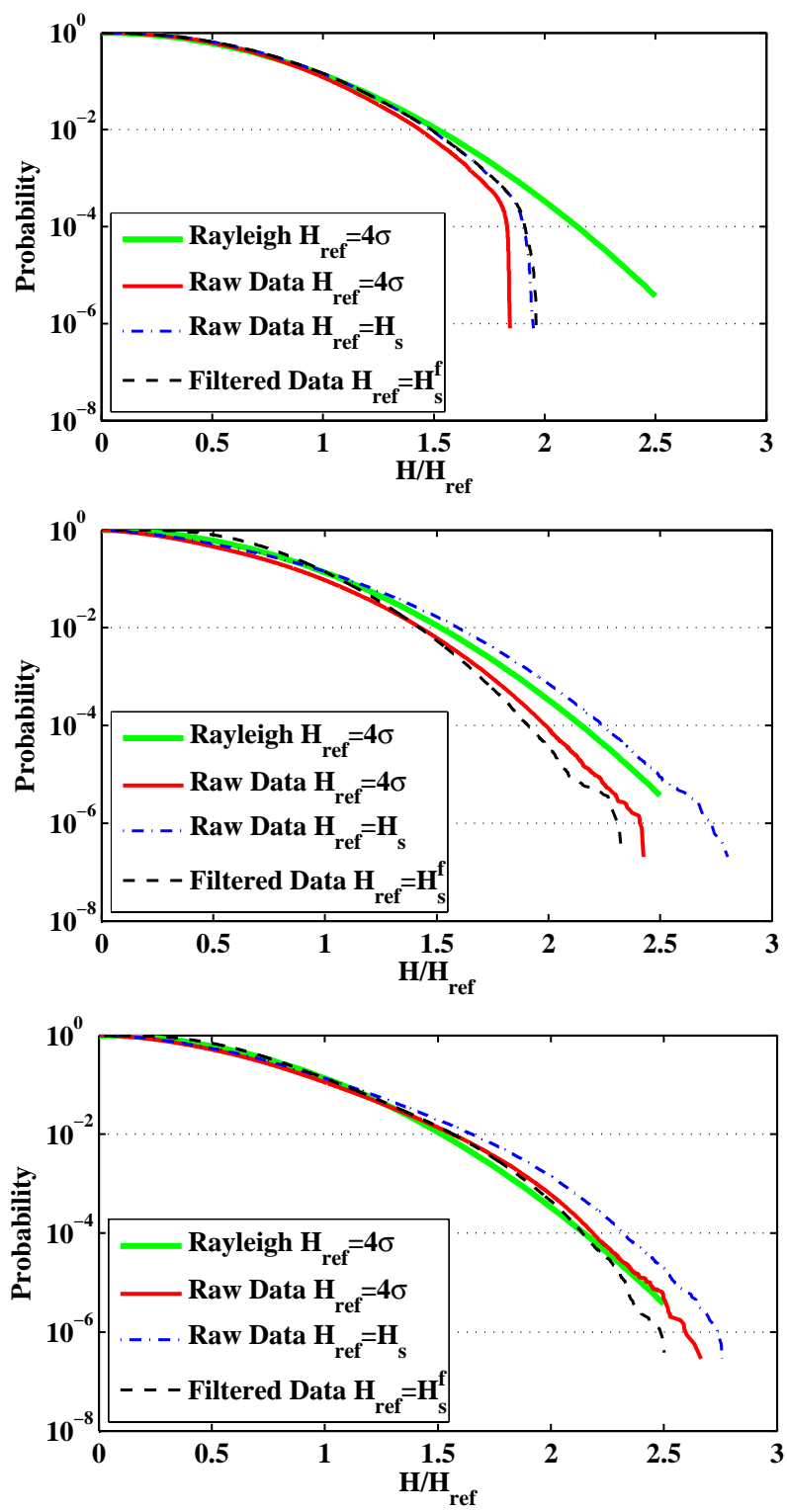

Fig. 4. Wave height exceedence probability distribution functions for conditions A simulated within the linear framework (case AL) (a) and by means of $\operatorname{HOSM}(M=6)(\mathbf{b})$, and for condition $E$ simulated by means of the HOSM $(M=3)$ (c) . The PDF are produced for the raw and filtered data; wave heights are normalized by different factors Href (see legend and discussion in the text). The theoretical Rayleigh distribution (thick line) is given for the reference.

We should mention that the statistics of simulated nonlinear waves deviates from the Gaussian one, and values of $H_{\mathrm{s}}$, calculated according to the general definition, differ from the value of $4 \sigma$, which leads to a different criterion for the rogue wave selection. For simplicity of selecting rogue waves, we use criterion (7) with $H_{\mathrm{s}}$ equal to $4 \sigma$, i.e., for rogue waves $H>8 \sigma$. In total about 800 rogue waves are found for the sea state A, and about 4500 waves for the more severe case E. 


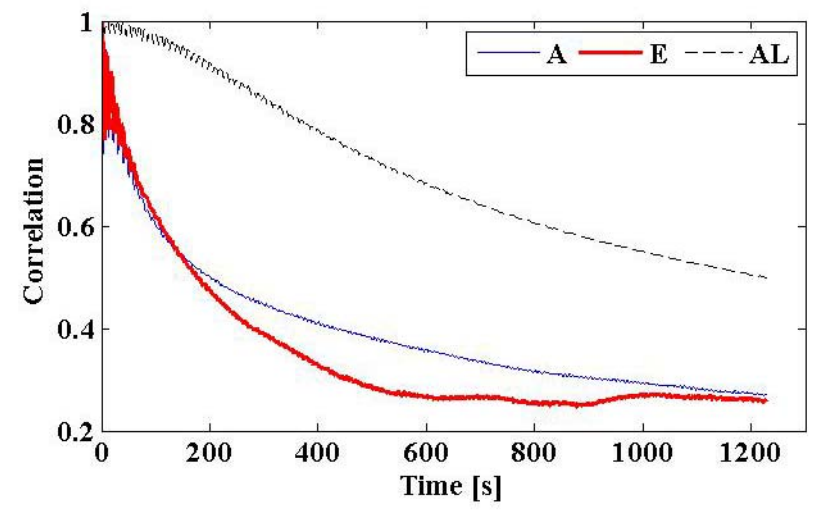

Fig. 5. Wave correlation $\mathrm{R}(0, \Delta \mathrm{t})$ for conditions $\mathrm{A}$ (thick solid line) and $\mathrm{E}$ (thin solid line) 9 and also for case $\mathrm{A}$, when the evolution is simulated within the linear theory, AL (dashed 10 line).

One of the aims of the present work is to consider the variety of shapes of rogue waves. To this end, individual waves must be singled out. Usually, this is performed by the zerocrossing approach, see e.g. (Holthuijsen, 2007). It is substantial that in the present case we deal with space series, but not time series. It is very well understood that in deep-water conditions waves have an effectively narrower frequency spectrum in comparison to the wavenumber spectrum. As a consequence, the portion of short-scale waves which can be distinguished on water surface is greater in the spatial domain. They look like ripples on the background of wind waves; many of them are recognized as individual waves according to the naive application of the zero-crossing approach. The large amount of small-scale weak-amplitude waves distorts statistical properties (such as typical wave length, significant wave height) greatly. To avoid this spurious effect, wave filtering was introduced. First, individual waves were separated following the basic zero-crossing approach (hereafter this selection of individual waves in spatial series will be referred to as raw data). If an individual wave happens to be significantly shorter than the peak wave length (shorter than $L_{0} / 5$, where $L_{0}=T_{0}^{2} g /(2 \pi)$ ), it is merged by the neighboring individual wave. Therefore, the number of individual waves after the filtering is smaller than the one in the raw data, and the significant wave height is, correspondingly, larger in the filtered data.

\subsection{Probability distribution functions}

We have already noted the difference between statistical data, used in this study, and that composed by conventional time series retrieved at a single point: not all individual waves (among about 2.5 million) are independent. Therefore, the direct comparison between the statistical results becomes impossible, and it is desirable to understand at least the main features of the difference.

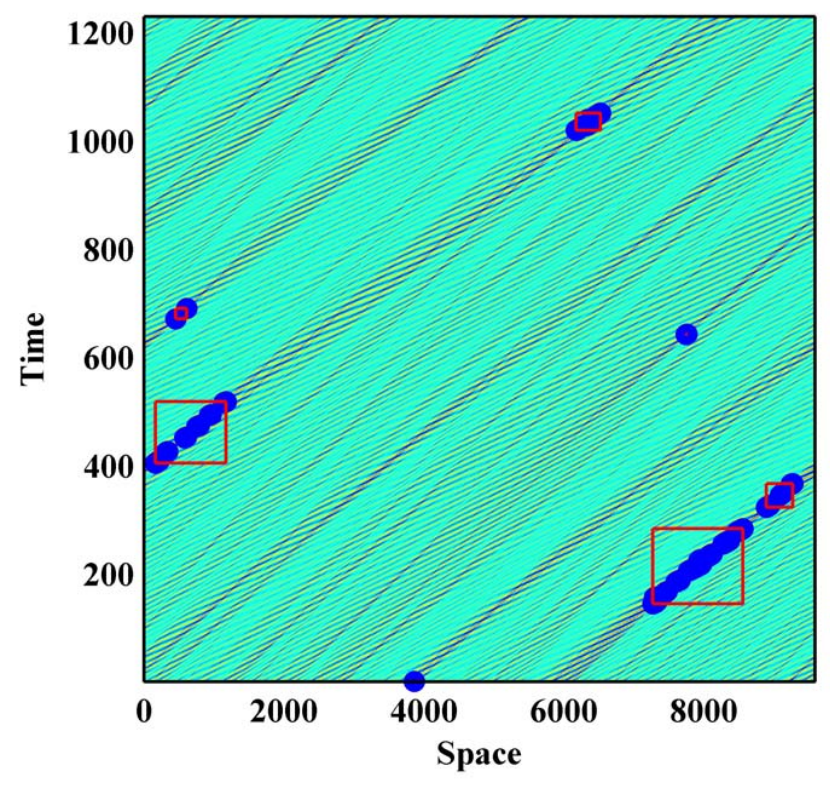

Fig. 6. Spatio-temporal sheet of the surface elevation. Markers indicate rogue waves, and rectangles assemble them into rogue events.

First, we employ the advantage of fully controllable numerical simulations, and conduct numerical simulations of 20 realizations for the initial condition A, when nonlinear effects are switched off totally (this numerical experiment will be hereafter referred to as AL), and then produce the exceedance probability distribution function on the basis of the 2.5 million individual waves. The result of processing is shown in Fig. 4a. The probability distribution functions are produced for the raw and filtered data, denoted as $H_{\mathrm{s}}$ and $H_{\mathrm{s}}^{f}$ respectively; inequality $H_{\mathrm{s}}^{f} \geq H_{\mathrm{s}}$ takes place. The significant wave heights are defined as a mean of significant heights of zero down-crossing waves and up-crossing waves. The horizontal axis in Fig. 4 shows the normalized height, where the normalization factor, $H_{\text {ref }}$, is given in the figure legend. The Rayleigh probability function, which follows from the linear theory for narrow band waves, is given for the reference by a thick line.

The first important observation is that no rogue waves occur in simulation $\mathrm{AL}$ at all, since all the curves in Fig. 4a

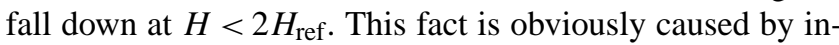
sufficient volume of the statistical ensemble. The number of narrow-banded individual waves in the ensemble, which results in the Rayleigh distribution for wave heights, exceeding $4 \sigma$, was estimated in Pelinovsky and Kokorina, (2002) to be about 10000 . Even a greater number of realizations should be simulated with the purpose of studying the rogue wave statistics in conditions AL. There is practically no difference between curves for $H_{\mathrm{ref}}=H_{\mathrm{s}}^{f}$ and $H_{\mathrm{ref}}=H_{\mathrm{s}}$. The tail of the curve for $H_{\text {ref }}=4 \sigma$ is somewhat lower than the one for $H_{\text {ref }}=H_{\mathrm{s}}$, which is because the value $4 \sigma$ exceeds significant 
wave height, and both the tails are lower than the Rayleigh function.

Figure 4b,c displays the results of nonlinear wave evolution. Appearance of long tails is evident, and waves exceeding $H>2 H_{\text {ref }}$ occur in all cases. The PDF's tails for the ensemble of non-filtered waves exceed theoretical curve when $H_{\text {ref }}=2 H_{\mathrm{s}}$, both in Fig. $4 \mathrm{~b}$ (moderately intense waves, A) and in Fig. 4c (severe wave conditions, E); for these cases $H_{\mathrm{S}}<\sim 4 \sigma$, similar to conditions AL. At the same time, the calculated PDF with scaling $H_{\text {ref }}=4 \sigma$ is lower than the Rayleigh curve in case A (Fig. 4b) and seems to be a bit higher than in case E (Fig. 4c). Note that the slightly different definition of the quantity $H_{\mathrm{s}}$ is able to change the positioning of the calculated curves with respect to the Rayleigh function.

The impact of the wave filtering, which sifts out shortscale waves, becomes very important in nonlinear simulations (Fig. 4b, c), in contrast to the linear case (Fig. 4a). Due to the filtering, the amount of small waves decreases greatly, $H_{\mathrm{s}}^{f}$ grows respectively, and the number of waves with $H>2 H_{\mathrm{s}}^{f}$ is much smaller than of waves with $H>2 H_{\mathrm{s}}$ or $H>8 \sigma$. As a result, the probability distribution function for filtered data falls below the Rayleigh function. We emphasize that in the present study the wave processing is performed for the space series, but not for time series. Because frequency wave spectrum of deep-water waves is about twice as narrow as the wavenumber spectrum, it is natural to expect that the effect of small-scale waves on the distribution function in the case of time series processing is less prominent.

The study of wave auto-correlation in time and space is illustrated in Fig. 5. The wave correlation is estimated with the help of the following function,

$$
R(t, \Delta t)=\max _{\Delta x} \frac{\int \eta(t, x) \eta(t+\Delta t, x+\Delta x) d x}{\sqrt{\int \eta^{2}(t, x) d x} \sqrt{\int \eta^{2}(t+\Delta t, x) d x}},
$$

where the integration is made over the spatial domain. For simplicity, function $R(0, \Delta t)$ is examined and is plotted in Fig. 5. Three curves are shown in the figure: the thin solid line corresponds to the case $\mathrm{A}$; the case $\mathrm{E}$ is marked with the thick solid line; the dashed curve displays the result of simulation, when the same initial condition as in case $\mathrm{A}$ is simulated within the linear framework (AL). Figure 5 shows very different pictures for the linear and nonlinear wave dynamics. It reports that the level of correlation decays much faster in the nonlinear case in comparison to the linear simulation. Sea states with relatively small waves and with steeper waves do not differ significantly.

According to the analysis, the effect of higher probability of high waves with respect to the linear theory, observed in the present simulations (see Fig. 4), is assumed by us to be twofold. First, nonlinearity is generally known to increase the likelihood of high waves; this effect was observed both in deep and shallow water conditions (Onorato et al., 2001, 2002; Pelinovsky and Sergeeva, 2006; and many others).

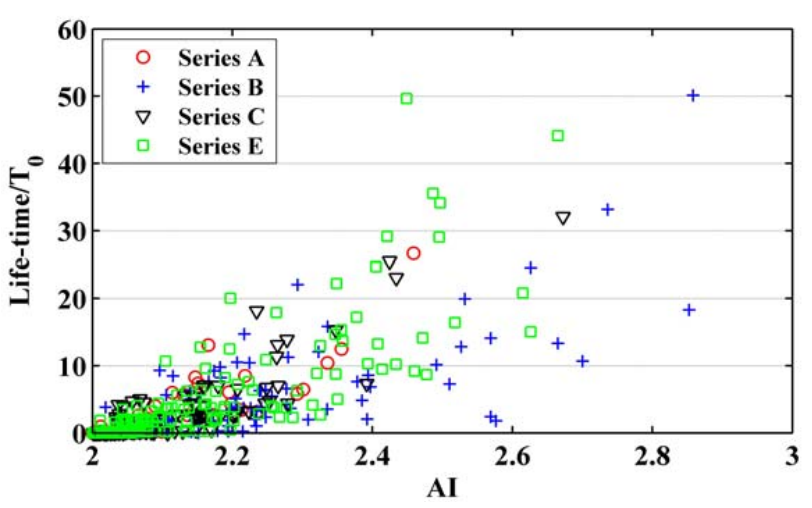

Fig. 7. Normalized lifetime of rogue events versus the abnormality index, AI.

Qualitatively this effect is in agreement with the formation of long-living nonlinear wave structures (coherent nonlinear wave groups in deep water, and solitary waves in shallow water). The second effect, which becomes vivid due to Fig. 5, is shortening of the wave correlation length, more waves among the 2.5 million become effectively independent due to nonlinear effects, and hence the volume of the statistical ensemble effectively increases, and higher waves may be found.

\subsection{Pictures of rogue waves and rogue events}

An example of the simulated $(x, t)$ sheet of surface elevations with detected rogue waves is given in Fig. 6. Filled circles mark extreme waves with $A I>2$. As it is described above, the spatial domain is periodic, and space series are analyzed.

To follow the evolution of a rogue wave, the wave height may get smaller than the threshold value (7), which specifies rogue waves for a short time, and may exceed the threshold soon after. Thus, such extreme waves in the course of its evolution satisfies criterion (7) in a discontinuous manner. It is natural to consider this sequence of extreme waves as a rogue event, even if the wave does not satisfy condition $A I>2$ at all instants of its lifetime. Therefore, individual waves, which satisfy the rogue wave criterion (7), are assumed to belong to one rogue event, if they are located in space and time not further from each other than 2.5 characteristic wave lengths and 2.5 characteristic wave periods.

In Fig. 6 clustered rogue events are shown by frames, which assemble rogue waves (filled circles). Very shortliving single rogue waves (of one wave period or less), and continuous and intermittent long-living rogue events (lasting for many wave periods) may be observed in the figure (note periodic boundary conditions for the simulated spatial domain.) Long-living rogue waves were found, in particular, in numerical simulations by Clamond and Grue (2002), Slunyaev and Sergeeva (2012), Slunyaev and Shrira (2013). An 


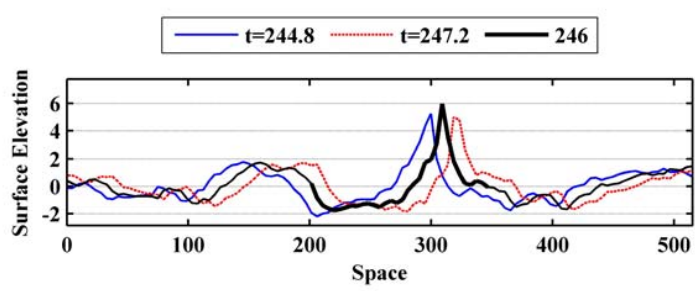

(a)

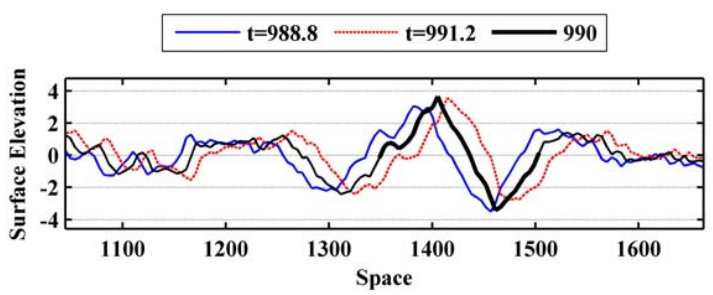

(b)

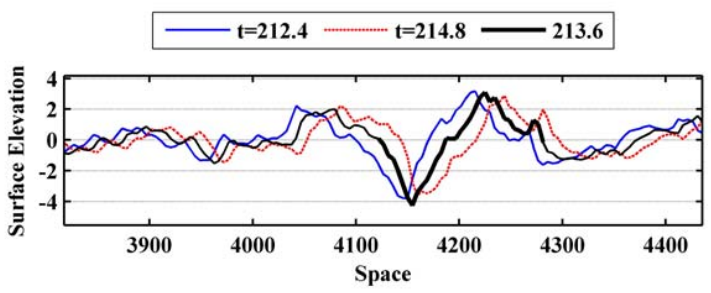

(c)

Fig. 8. Examples of rogue wave profiles (bold lines), and surface elevations at consecutive time points: single crest (a), sign-variable wave (b), deep hole (c).

even longer rogue event might be distinguished in the figure (from $150 \mathrm{~s}$ to about $550 \mathrm{~s}$ ), if a longer separation between the individual rogue waves is allowed.

Of course, the life time of a rogue event can be significantly longer than for continuous rogue waves. The spread of values of rogue event's lifetimes versus the abnormality index is pictured in Fig. 7 for series A, B, C and E. The figure claims that rogue events may last for a few dozen characteristic wave periods, up to about $10 \mathrm{~min}$. This lifetime significantly exceeds some other estimations on the rogue wave lifetime, including the results of our previous studies, where the lifetimes were estimated to be up to about $1.5 \mathrm{~min}$. Figure 7 exhibits the trend that more intense waves live for a longer time.

Typical shapes of rogue waves observed in the numerical simulations are shown in Fig. 8. The bold lines depict the rogue waves, how they are segregated by the present processing. Most of rogue waves correspond to high crests (Fig. 8a) or skew-symmetric surfaces (Fig. 8b); besides, extreme waves with deep troughs ("holes in the sea") are also found (Fig. 8c). Thin lines in Fig. 8 correspond to surface elevations at consecutive time instants close to the moment of the rogue wave occurrence.
Two approaches to selecting wave heights are usually applied to processing wave records: zero down-crossing or zero up-crossing (e.g., Holthuijsen, 2007). Note that an upcrossing wave in a time series becomes a down-crossing wave when its momentary shape is considered as a function of a coordinate (and the coordinate axis is chosen along the wave propagation). In the present analysis, a rogue wave owns an extreme vertical excursion between the wave crest and the wave trough; each crest and trough, localized according to the zero-crossing method, constitute the rogue wave, as shown in Fig. 8 by bold lines. A rogue wave may be either up- or down-crossing, depending on the mutual location of the wave crest and trough constituting the rogue wave. Waves in Fig. 8 represent space series of waves which propagate to the right. Extreme waves in Fig. 8a, c (given by the bold line) are zero down-crossing waves, and will be referred to as extreme rear slope waves. The spatial record of an extreme wave, given in Fig. 8b, is an up-crossing wave and is represented by an extreme frontal slope.

A variety of rogue wave geometries found in the numerical simulations is illustrated in Figs. 9 and 10. Figure 9 displays the relation between the ratio of crest amplitude to trough amplitude (vertical axis) versus the ratio of crest length to trough length (horizontal axis) for wave conditions A (Fig. 9a) and E (Fig. 9b). The distinction is made between the cases when the rogue wave has a huge frontal slope (shown with circles) or an extreme rear slope (shown with crosses). In Fig. 9 all the rogue waves found in 20 realizations for cases $\mathrm{A}$ and $\mathrm{E}$ are collected. Figure 9 proves that extreme waves with high crests are prevalent in comparison with extremely deep troughs. Higher crests are shorter in length.

The proportion between rogue waves of different shapes is displayed in Fig. 10 for sea states A and E respectively. Four kinds of rogue waves are distinguished regarding the vertical asymmetry and orientation of the wave (extreme frontal or rear slope). Both panels in Figs. 10a,b claim prevalence of waves with high crests ("positive" waves, 85-95\%), while waves with deep troughs ("negative" waves) are much less in number, agreeing with the results in Fig. 9.

For less intense waves (Series A, Fig. 10a) the proportion between up-crossing and down-crossing rogue waves is about the same, whereas in the steeper wave case (Series E, Fig. 10b) the total number of recognized rogue waves is much greater, and the number of rogue waves with extreme rear slopes $(61 \%)$ is significantly larger than with extreme fronts (34\%), thus in the majority of cases a ship should experience a very high wave crest first, and then a subsequent deep trough. This picture is opposite to the one known in relation to regions with strong opposite currents (Mallory, 1974; Lavrenov, 1998), when a deep trough precedes a high crest (a huge wave front), but agrees very well with the in situ measurements by Pinho et al. (2004), where 276 cases of rogue waves result in the proportion between waves with large rear and frontal slopes as 197:108. 


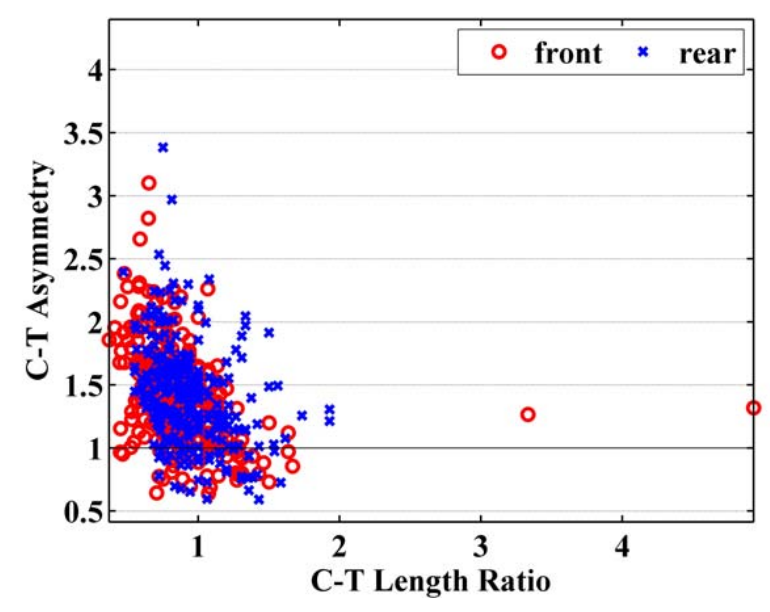

a)

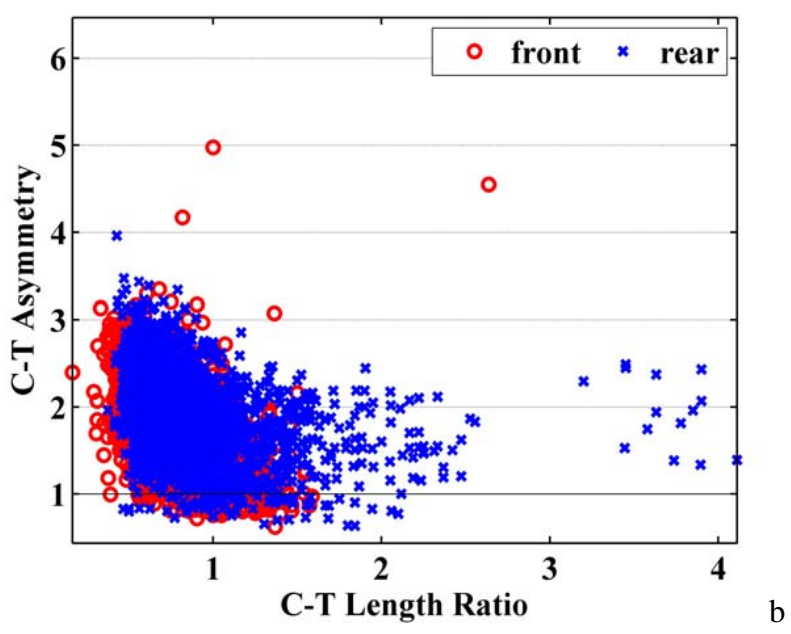

Fig. 9. Relation between vertical wave asymmetry (crest amplitude to trough amplitude) and horizontal asymmetry (crest length to trough length) for two types of rogue waves (circles show cases with extreme wave fronts, while crosses denote extreme rear slopes) for series A (a) and E (b).

\section{Extreme kinematics of waves versus kinematics of extremely high waves}

Establishing the relation between the extreme surface elevation and extreme wave kinematics is an important issue. Conventionally the rogue wave criterion is based on the amplitude characteristics (7). The reason for that is evident: although waves are in fact a complicated phenomenon represented by the movement of fluid particles, the surface elevation is much easier to observe and to register.

Through the performed simulations, a large amount of information on wave kinematics is available, enabling us to conduct a kind of statistical study on rogue wave kinematics characterizing particular sea states. The employed numerical

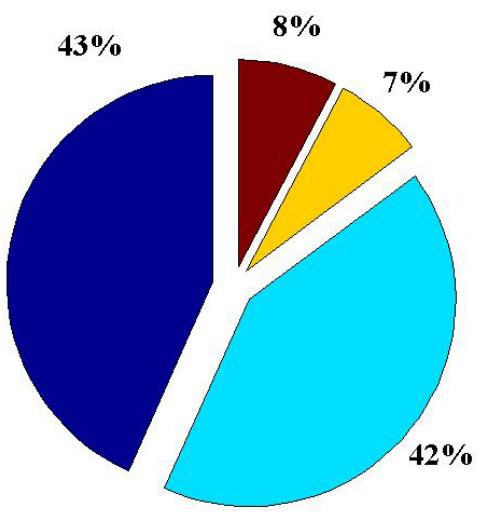

a)

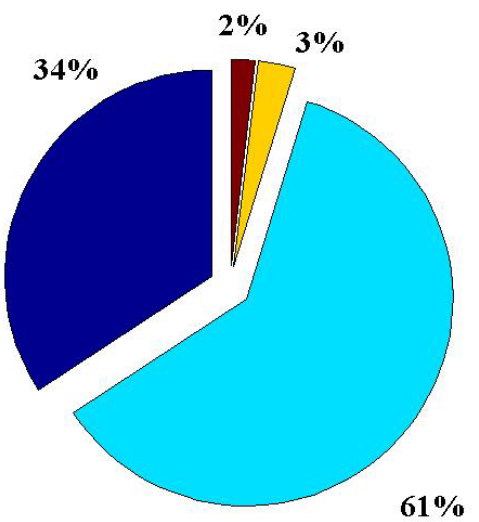

b)

Fig. 10. The proportions of waves of different types in the total mass of rogue waves for series A (a) and E (b).

model cannot simulate wave breaking, hence breaking waves are beyond the scopes of the present analysis.

In this section we consider the relation between extreme velocities in the simulated wave fields, and velocities of rogue waves (the latter are singled out on the basis of the wave height criterion (7)) and address the question: do rogue waves manifest faster velocities?

The Eulerian horizontal and vertical velocities of the water surface, $V_{x}$ and $V_{z}$ are defined by

$$
\left.V_{x} \equiv \frac{\partial \phi}{\partial x}\right|_{z=\eta}=\frac{\Phi_{x}-\eta_{t} \eta_{x}}{1+\eta_{x}^{2}},\left.V_{z} \equiv \frac{\partial \phi}{\partial z}\right|_{z=\eta}=\frac{\eta_{t}+\Phi_{x} \eta_{x}}{1+\eta_{x}^{2}}
$$

The formula for $V_{z}$ results from (1), and the formula for $V_{x}$ comes from the identity $\Phi_{x}=\phi_{x}+\eta_{x} \phi_{z}$ at $z=\eta$.

A simulation of one irregular wave realization results in $2048 \times 2048$ values of velocities (9). They are plotted in 


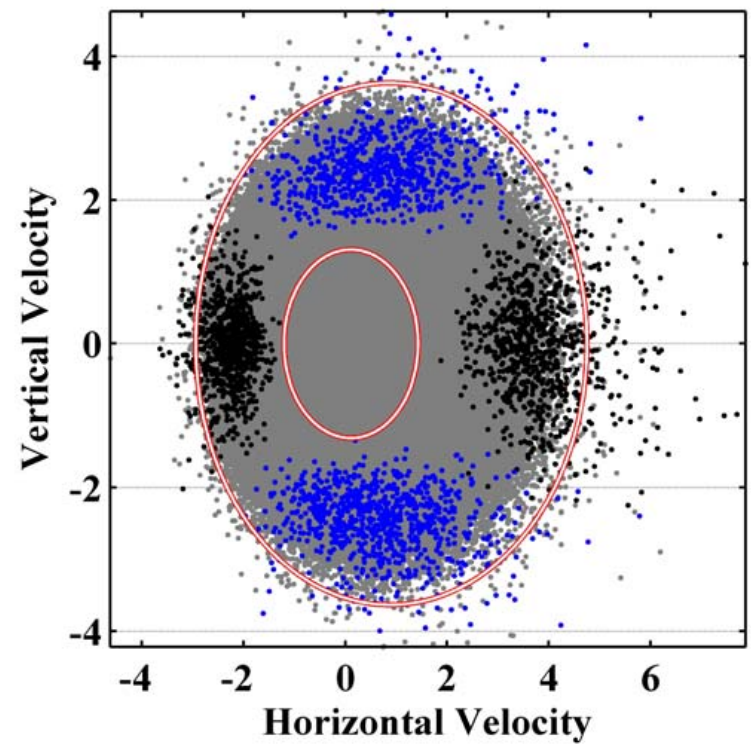

a)

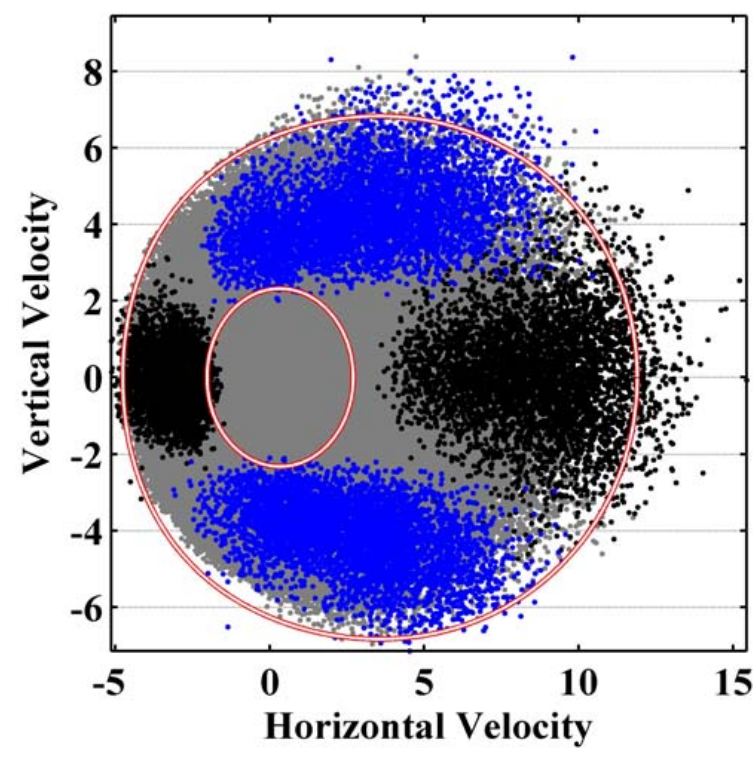

b)

Fig. 11. Grey points are all the values of vertical versus horizontal surface velocities in the $(\mathrm{x}, \mathrm{t})$ fields (5 realizations are used). Black points are the maximum horizontal and blue points are the maximum vertical velocities attained by the detected rogue waves (all 20 realizations are used to plot these values): (a) Series A, (b) Series E. Contours correspond to the values for the 3rd order Stokes wave solution of the heights $H_{\mathrm{S}}$ (the smaller contour) and $\mathrm{H}_{\max }$ (the larger contour).

diagrams in Figs. 11 with grey points $\left(V_{x}\right.$ versus $\left.V_{z}\right)$ for five realizations of sea states A (Fig. 11a) and E (Fig. 11b). The extreme values of horizontal (black points) and vertical (blue points) velocities, which are attained by the segregated rogue waves (see examples given by bold lines in Fig. 8), are shown for all 20 realizations, simulated for conditions $\mathrm{A}$ and $\mathrm{E}$.

For reference, velocities at the water surface for the 3rd order Stokes wave solution are shown in Figs. 11 (contours) according to formulas, see e.g. (Fenton, 1985; Slunyaev, 2005)

$$
\begin{aligned}
& V_{x}=\sqrt{k_{0} g} A\left(1-\frac{1}{8} k_{0}^{2} A^{2}\right) \exp \left(k_{0} \eta\right) \cos \theta, \\
& V_{z}=\sqrt{k_{0} g} A\left(1-\frac{1}{8} k_{0}^{2} A^{2}\right) \exp \left(k_{0} \eta\right) \sin \theta, \\
& \eta=A \cos \theta+\frac{k_{0}}{2} A^{2} \cos 2 \theta+\frac{3}{8} k_{0}^{2} A^{3} \cos 3 \theta .
\end{aligned}
$$

Here $A$ is the wave amplitude, and $0<\theta<2 \pi$ is the wave phase; $k_{0}=\omega_{0}^{2} / g$ is the carrier wavenumber, and $\omega_{0}$ comes from the JONSWAP spectrum (5). The contours are plotted in Figs. 11 for two wave intensities: (i) when the wave height is equal to the significant wave height, $H_{\mathrm{S}}$ (then $A=H_{\mathrm{s}} / 2$ $3 / 64 k_{0}^{2} H_{\mathrm{s}}^{3}$, the smaller contour); and (ii) for the maximum wave height registered in the simulated wave fields, $H_{\max }$ (then $A=H_{\max } / 2-3 / 64 k_{0}^{2} H_{\max }^{3}$, the larger contour).

The larger contour corresponds to the highest possible velocity of surface waves as if they were described by the 3rd order Stokes wave solution (10-12). The area inside the contour corresponds well to the major amount of velocities in the both cases, Series A and E. The inner contour allocates the range of wave velocities with heights smaller than $H_{\mathrm{s}}$; this area of velocities $\left(V_{x}, V_{z}\right)$ is practically free of rogue waves. The 5th order Stokes wave solution for surface velocities does not significantly change the picture shown in Fig. 11a (Series A), it is quite similar to the 3 rd order velocities. For severer Series E the value of $H_{\max }$ seems to be so large that the asymptotic solution for wave velocities does not converge well.

Asymmetries of the vertical and horizontal velocity fields are well pronounced, the velocities, which correspond to rogue waves, have qualitatively similar distribution asymmetry. The asymmetries strengthen for a steeper wave sea state.

Figure 11 proves the inability of the high-order Stokes wave theory to describe the rogue wave kinematics, although the major part of velocities attained by simulated rogue waves lies within the ranges, which correspond to the highest registered wave. Some "blind" areas in Fig. 11 are evident (where only grey points exist), which correspond to intense velocities (they may even exceed the limit set by the Stokes wave solution) and at the same time to relatively small wave amplitudes (rogue waves with these fluid velocities are not found in the records). On the other hand, some rogue waves exhibit quite moderate values of velocities.

The analysis of velocities and accelerations under crests of steep waves was performed in (Grue et al., 2003; Grue 


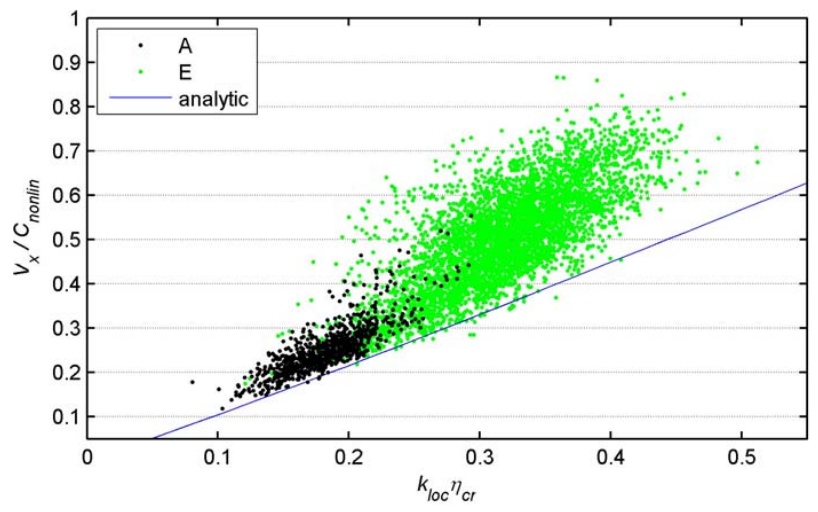

Fig. 12. Scaled horizontal velocities $V_{x} / C_{\text {nonlin }}$ versus scaled crest amplitudes $\mathrm{k}_{\text {loc }} \mathrm{n}_{\text {or }}$ found in rogue waves in numerical simulations of sea states $\mathrm{A}$ and $\mathrm{E}$ (points). The solid curve gives the analytic estimation from the asymptotic weakly nonlinear theory.

and Jansen, 2006, 2012). In particular, profiles of horizontal velocities and maximal horizontal velocities with respect to the wave speed were considered. The exponential profile was found to describe the situation very well. Two characteristic velocity scales were suggested, the linear phase velocity, $\mathrm{C}_{\text {lin }} \equiv A \sqrt{k_{0} g}$ (cf. $(10,11)$ ), and the velocity which takes into account a small nonlinear correction to frequency, $C_{\text {nonlin }} \equiv A \sqrt{k_{0} g}\left(1+k_{0}^{2} A^{2}\right)$. Grue et al. (2003) overviewed the maximum horizontal fluid velocities reported by different researchers. They found that the fluid velocities were generally smaller than the nonlinear wave celerity, $C_{\text {nonlin }}$, but seemed to be strongly dependent on wave types (depth conditions, breaking situations) that sometimes could exceed $C_{\text {nonlin. }}$.

In this paper we consider only the fluid velocities at the water surface. In Fig. 12 the maximum velocity value $V_{x} / C_{\text {nonlin }}$ for each rogue wave is indicated by a symbol versus the dimensionless rogue wave crest amplitude, $k_{\text {loc }} \eta_{c r}$.; only the waves which satisfy the rogue wave criterion are concerned. Sea states A and E are shown by black and green points, respectively. Here the local wavenumber, $k_{\text {loc }}$, is determined according to the zero-crossing wave length, and $\eta_{c r}$ is related with $A$ by formula (12), when $\theta=0$. The values of $k_{\text {loc }} \eta_{c r}$ in Fig. 12 are up to 0.29 for the sea state A, and up to 0.51 for the sea state $\mathrm{E}$; the corresponding maximum scaled velocities are up to 0.55 and 0.87 . For values $V_{x} / C_{\text {lin }}$, the clouds of data are significantly more spread (not shown).

According to Fig. 11, formulas (10-12) are expected to describe the major part of the velocity data rather well. For the maximal registered values of $k_{\text {loc }} \eta_{c r}$, which are shown in Fig. 12, formulas (10-12) result in estimations $V_{x} / C_{\text {nonlin }}=$ 0.32 for case $\mathrm{A}$, and $V_{x} / C_{\text {nonlin }}=0.60$ for case $\mathrm{E}$; the corresponding analytic curve is plotted in Fig. 12 for reference. This estimation obviously limits the data of numerical experiments from below. Actual velocities may exceed this estimation substantially, but they are still always less than $C_{\text {nonlin. }}$ The numerical simulations reported in (Grue et al., 2003) seem to agree better with the theoretical curve. It is worth mentioning that the way in which the local wavenumber is estimated is different in Grue et al. (2003) and in the present study. In Grue et al. (2003) the trough-to-trough distance around an intense crest in time series is estimated, while we use the zero-crossing wave length around an extreme elevation drop is space series.

\section{Conclusions}

In the paper we present the approach to a more extensive use of data on rogue wave dynamics, statistics, and kinematics, which becomes available through stochastic simulations of irregular waves.

In this study waves characterized by the JONSWAP spectrum are simulated by means of the strongly nonlinear algorithm for solving the Euler equations, the HOSM. Two sea states are considered in more detail - of moderately high waves and steeper waves. For each sea state 20 realizations are simulated, each results in $10 \mathrm{~km} \times 20$ min sheets of data with good resolution of the surface elevation, fluid velocity, etc.

The accumulated data may be used for generating temporal or spatial wave records, and then used for verification and adjustment of processing techniques. The availability of full spatio-temporal information about the wave field provides an opportunity to clarify the dispersion relation for nonlinear waves, where wave components (free, bound and counterdirectional modes) are naturally separated (as discussed in Slunyaev and Sergeeva, 2012).

In the present study the main interest is focused on characteristics of rogue waves which occur in the simulated fields. The simulated data contains a number of rogue waves available for analysis. Space series are used, because of the peculiarity of periodic spatial boundary conditions. Spatial time series (one- and two-dimensional) of the surface elevation, which are recorded in laboratory and in situ conditions are becoming available nowadays due to the development of new measuring techniques. Hence, the direct comparison between the theoretical simulation and measurements becomes potentially possible.

The analysis of characteristics of individual rogue waves, found in our simulations, shows the variety of their shapes and appearances. Most of the simulated rogue waves have very high crests and shallow long troughs, or sign-variable shapes. "Holes in the sea", characterized by deep troughs, are found in the simulation data as well. In severe conditions, the predominance of rogue waves with extreme rear slopes is discovered compared to rogue waves with high fronts.

The employed approach allows for the observation of a full picture of the rogue wave propagation. Some of extreme waves appear suddenly and live for a short time, less then a 
wave period. Large amount of rogue waves' heights oscillate with time and exceed the formal criterion of a rogue wave, $H>2 H_{\mathrm{s}}$, repeatedly, and thus may be considered as rogue events which can live significantly longer than the wave, which satisfies the rogue wave amplitude criterion continuously. As a result, the lifetime of extremely high wave events varies from several seconds to tens of characteristic wave periods.

Water velocities at the surface are considered in relation to the rogue wave phenomenon. The velocities of extreme waves are compared versus extreme kinematics of all waves and with predictions of the 3-order and 5-order nonlinear theories for Stokes waves. The difference between the kinematics of extremely high waves and the extreme kinematics is emphasized, whereas the significant amount of data well correspond to each other. Rogue waves are typically characterized by large values of velocities, but high velocities do not necessarily correspond to rogue waves, and rogue waves may be characterized by moderate fluid velocities. The maximal horizontal fluid velocities at the surface of rogue waves may be rather well parametrized over the wave velocity, which takes into account the nonlinear frequency correction.

Acknowledgements. The research is supported by the EC's Seventh Framework Programme FP7-SST-2008-RTD-1 project EXTREME SEAS - Design for Ship Safety in Extreme Seas under grant agreement No. 234175, RFBR grants 11-02-00483, 11-05-92002, 12-05-33087 and 11-05-00216. A. Sergeeva acknowledges support from the Volkswagen Foundation and MK-5222.2013.5. A. Slunyaev acknowledges support from the EC's Programme FP7PEOPLE-2009-IIF under grant agreements No. 254389/909389.

Edited by: E. Pelinovsky

Reviewed by: two anonymous referees

\section{References}

Annenkov, S. Y. and Shrira, V. I.: Evolution of kurtosis for wind waves, Geophys. Res. Lett., 36, L13603, doi:10.1029/2009GL038613, 2009.

Chalikov, D.: Statistical properties of nonlinear one-dimensional wave fields, Nonlin. Processes Geophys., 12, 671-689, doi:10.5194/npg-12-671-2005, 2005.

Chalikov, D.: Freak waves: Their occurrence and probability, Phys. Fluids, 21, 076602, doi:10.1063/1.3175713, 2009.

Christou, M. and Ewans, K.: Examining a comprehensive dataset containing thousands of freak wave events. Part 1 - description of the data and quality control procedure, Proceedings of 30th International Conference on Ocean, Offshore and Arctic Engineering (OMAE-2011), OMAE2011-50168 doi:10.1115/OMAE201150168, 2011.

Clamond, D., Francius, M., Grue, J., and Kharif, C.: Long time interaction of envelope solitons and freak wave formations, Eur. J. Mech. B/Fluids, 25, 536-553, 2006.
Clamond, D. and Grue, J.: Interaction between envelope solitons as a model for freak wave formations, C. R. Mecanique, 330, 575580, 2002.

Dommermuth, D. and Yue, D. K. P.: A high-order spectral method for the study of nonlinear gravity waves, J. Fluid Mech., 184, 267-288, 1987.

Dysthe, K. B., Trulsen, K., Krogstad, H. E., and Socquet-Juglard, H.: Evolution of a narrow-band spectrum of random surface gravity waves, J. Fluid. Mech., 478, 1-10, 2003.

Dysthe, K. B., Krogstad, H. E., and Müller, P.: Oceanic rogue waves, Annu. Rev. Fluid. Mech., 40, 287-310, 2008.

Fenton, J. D.: A fifth-order Stokes theory for steady waves, J. Waterway, Port, Coastal Ocean Eng., 111, 216-234, 1985.

Gramstad, O. and Trulsen, K.: Influence of crest and group length on the occurrence of freak waves, J. Fluid. Mech., 582, 463-472, 2007.

Grue, J., Clamond, D., Huseby, M., and Jensen, A.: Kinematics of extreme waves in deep water, Appl. Ocean Res., 25, 355-366, 2003.

Grue, J. and Jensen, A.: Experimental velocities and accelerations in very steep wave events in deep water, Eur. J. Mech. B/Fluids, 25, 554-564, 2006.

Grue, J. and Jensen, A.: Orbital velocity and breaking in steep random gravity waves, J. Geophys. Res., 117, C07013, doi:10.1029/2012JC008024, 2012.

Janssen, P. A. E. M.: Nonlinear four wave interactions and freak waves, J. Geophys. Oceanogr., 33, 863-884, 2003.

Holthuijsen, L. H.: Waves in oceanic and coastal waters, Cambridge Univ. Press, 2007.

Kharif, C., Pelinovsky, E., and Slunyaev, A.: Rogue Waves in the Ocean, Berlin Heidelberg, Springer-Verlag, 2009.

Lavrenov, I.: The wave energy concentration at the Agulhas current of South Africa, Nat. Hazards, 17, 117-127, 1998.

Mallory, J. K.: Abnormal waves on the south-east of South Africa, Int. Hydrog. Rev., 51, 99-129, 1974.

Mori, N., Onorato, M., Janssen, P. A. E. M., Osborne, A. R., and Serio, M.: On the extreme statistics of long-crested deep water waves: Theory and experiments, J. Geophys. Res., 112, C09011, doi:10.1029/2006JC004024, 2007.

Onorato, M., Osborne, A. R., Serio, M., and Bertone, S.: Freak waves in random oceanic sea states, Phys. Rev. Lett., 86, 58315834, 2001.

Onorato, M., Osborne, A. R., and Serio, M.: Extreme wave events in directional, random oceanic sea states, Phys. Fluids, 14, L25L28, 2002.

Onorato, M., Osborne, A. R., Serio, M., and Cavaleri, L.: Modulational instability and non-Gaussian statistics in experimental random water-wave trains, Phys. Fluids, 17, 078101, doi:10.1063/1.1946769, 2005.

Onorato, M., Waseda, T., Toffoli, A., Cavaleri, L., Gramstad, O., Janssen, P. A., Kinoshita, T., Monbaliu, J., Mori, N., Osborne, A. R., Serio, M., Stansberg, C. T., Tamura, H., and Trulsen, K. M.: Statistical properties of directional ocean waves: the role of the modulational instability in the formation of extreme events, Phys. Rev. Lett., 102, 114502, doi:10.1103/PhysRevLett.102.114502, 2009.

Pelinovsky, E. and Kokorina, A.: The applicability of the Kortewegde Vries equation for description of the statistics of the freak waves, J. Korean Soc. Coastal Ocean Engn., 14, 308-318, 2002. 
Pelinovsky, E. and Sergeeva, A.: Numerical modeling of the KdV random wave field, Eur. J. Mech. B/Fluid, 25, 425-434, 2006.

Pinho, U. F., Liu, P. C., and Ribeiro, C. E. P.: Freak Waves at Campos Basin, Brazil, Geofizika, 21, 53-67, 2004.

Shemer, L. and Sergeeva, A.: An experimental study of spatial evolution of statistical parameters of unidirectional narrowbanded random wave field, J. Geophys. Res., 114, C01015, doi:10.1029/2008JC005077, 2009.

Shemer, L., Sergeeva, A., and Liberzon, D.: Effect of the initial spectral shape on spatial evolution of the statistics of unidirectional nonlinear random waves, J. Geophys. Res., 115, 12039, doi:10.1029/2010JC006326, 2010a.

Shemer, L., Sergeeva, A., and Slunyaev, A.: Applicability of envelope model equations for simulation of narrow-spectrum unidirectional random field evolution: experimental validation, Phys. Fluids, 22, 016601, doi:10.1063/1.3290240, 2010b.

Slunyaev, A. V.: A high-order nonlinear envelope equation for gravity waves in finite-depth water, J. Experiment. Theor. Phys., 101, 926-941, 2005.

Slunyaev, A. V. and Sergeeva, A. V.: Stochastic simulation of unidirectional intense waves in deep water applied to rogue waves, JETP Letters, 94, 779-786, 2011.

Slunyaev, A. V. and Sergeeva, A. V.: Numerical simulations and analysis of spatio-temporal fields of rogue waves, Fundament. Appl. Hydrophys., 5, 24-36, 2012 (in Russian).
Slunyaev, A., Didenkulova, I., and Pelinovsky, E.: Rogue Waters, Contemporary Phys., 52, 571-590, 2011.

Slunyaev, A. V. and Shrira, V. I.: On the highest non-breaking wave in a group: fully nonlinear water wave breathers vs weakly nonlinear theory, in revision, J. Fluid Mech., 2013.

Socquet-Juglard, H., Dysthe, K. B., Trulsen, K., and Krogstad, H. E.: Probability distributions of surface gravity waves during spectral changes, J. Fluid Mech., 542, 195-216, 2005.

Tanaka, M.: A method of studying nonlinear random field of surface gravity waves by direct numerical simulation, Fluid Dyn. Res., 28, 41-60, doi:10.1016/S0169-5983(00)00011-3, 2001.

Trulsen, K.: Weakly nonlinear and stochastic properties of ocean wave fields: application to an extreme wave event, In: Waves in geophysical fluids: Tsunamis, Rogue waves, Internal waves and Internal tides, edited by: Grue, J. and Trulsen, K., CISM Courses and Lectures No. 489, Springer Wein, New York, 2006.

West, B. J., Brueckner, K. A., Janda, R. S., Milder, D. M., and Milton, R. L.: A new numerical method for surface hydrodynamics, J. Geophys. Res., 92, 11803-11824, 1987.

Zakharov, V.: Stability of periodic waves of finite amplitude on a surface of deep fluid, J. Appl. Mech. Tech. Phys., 2, 190-194, 1968. 